\title{
Beyond Hemoglobin: When and How to Work Up Possible Polycythemia Vera
}

\author{
Gene Shaw, MD and Richard Berg, MS
}

Background: World Health Organization (WHO) 2017 diagnostic criteria for hemoglobin levels in polycythemia vera (PV) were lowered from $185 \mathrm{~g} / \mathrm{L}$ to $165 \mathrm{~g} / \mathrm{L}$ for men and from $165 \mathrm{~g} / \mathrm{L}$ to $160 \mathrm{~g} / \mathrm{L}$ for women, but these cutoffs were not designed for screening.

Objectives: The primary aim of this study was to assess the value of laboratory and clinical parameters in deciding whether to further pursue a diagnosis of PV. A secondary aim was to explore the diagnostic utility of bone marrow morphology.

Methods: We evaluated clinical and laboratory parameters that may be useful when considering further diagnostic work-up, emphasizing PV vs. secondary erythrocytosis (SE). We classified 200 patients with JAK2 V6I7F testing using WHO criteria.

Results: Patients with myeloproliferative neoplasms (MPN) were rarely under age 40 and uncommonly obese (BMI $\left.\geq 30 \mathrm{~kg} / \mathrm{m}^{2}\right)$. Current smoking history favored $\mathrm{SE}$, and these patients rarely had a platelet count $\geq 450 \times 10^{3} /$ uL. Laboratory parameters suggesting greater PV likelihood were: $\mathrm{RBC}>6.8 \times 10^{6}$ for men or $>5.9 \times 10^{6}$ for women; low erythropoietin; and low MCV or low ferritin. Bone marrow morphology (available in III cases) was generally more cellular in PV vs. SE and assessed disease progression.

Conclusions: Readily accessible clinical and laboratory data can assist in considering a PV workup, and a possible diagnostic algorithm is presented. These preliminary findings warrant larger studies to develop a more formal PV-risk scoring system with optimal cutoffs and weighting.

Keywords: Polycythemia vera; Secondary erythrocytosis; Myeloproliferative neoplasms; JAK2 mutations; Erythropoietin

$\mathrm{T}$ The World Health Organization (WHO) diagnostic criteria for myeloproliferative neoplasms (MPN), including polycythemia vera (PV), were formally revised in 2017., ${ }^{1,2}$ To distinguish "masked" $P V$ from secondary erythrocytosis (SE), the major criterion for hemoglobin $(\mathrm{Hb})$ level in men was significantly decreased from $185 \mathrm{~g} / \mathrm{L}$ to 165 $\mathrm{g} / \mathrm{L}$, while the $\mathrm{Hb}$ level for women was slightly lowered from $165 \mathrm{~g} / \mathrm{L}$ to $160 \mathrm{~g} / \mathrm{L} .{ }^{3}$ Janus kinase 2 (JAK2) mutations (either JAK2 V617F in exon 14 or rarely mutations in exon 12) are present in virtually all PV patients. These mutations were originally included in the 2008 WHO criteria and have been retained as one of three major criteria. Bone marrow (BM)

Corresponding Author: Gene R. Shaw, MD, Department of Lab/Pathology, Marshfield Clinic, 1000 N. Oak Avenue, Marshfield,WI 54449

Tel: 7|5-22I-6I00, Email: shaw.gene@marshfieldclinic.org morphology has been elevated from a minor to a major criterion that emphasizes hypercellularity and pleomorphic megakaryocytes. ${ }^{4}$ Low serum erythropoietin (EPO) level is now the sole minor criterion. ${ }^{5}$

However, in clinical practice the diagnostic process is sequential, usually with abnormalities first noted in the complete blood count (CBC), which are then correlated with the clinical history and physical exam. The new WHO $\mathrm{Hb}$ criteria were not designed for general screening and are clearly too low for that purpose. ${ }^{6-8}$ The red blood cell count (RBC) may be underutilized. ${ }^{9}$ In addition, iron deficiency

Received: January 17, 2019

Revised: June 13, 2019

2nd revision: August I, 2019

Accepted: August 20, 2019 
may raise suspicion for $\mathrm{PV},{ }^{10}$ and the role of EPO warrants further investigation. ${ }^{11,12}$ Key clinical parameters include smoking history and body mass index (BMI); the latter is often associated with obstructive sleep apnea (OSA). If there is sufficient clinical suspicion for PV, JAK2 molecular testing is generally the next diagnostic step.

The primary aim of the current study was to assess the value of readily available laboratory and clinical parameters in making an informed decision on whether to further pursue a diagnosis of PV. A secondary aim was to explore the diagnostic utility of BM morphology.

\section{Materials and Methods}

Following Institutional Review Board approval, 200 cases of suspected MPN, other than chronic myeloid leukemia, that occurred after 2008 (when JAK2 testing became widely available) in the Marshfield Clinic system were identified. The following inclusion criteria were used: age 18 years or older, a JAK2 V617F mutation test result, and available clinical follow-up information. An exclusion criterion was $\geq$ $5 \%$ blasts in the BM at the time of MPN diagnostic evaluation.

The white blood cell (WBC) count at the time of initial diagnostic evaluation was recorded along with the highest values on or prior to that date for $\mathrm{Hb}, \mathrm{RBC}$, and platelets along with the lowest values for MCV, EPO, and ferritin. Transiently abnormal values due to a clinical condition (eg, blood loss) were not included.

For BM evaluation, the following data were obtained from the medical record: cellularity, myeloid:erythroid (M:E) ratio, megakaryocyte morphology (increased and/or abnormal), presence or absence of stainable iron, reticulin fibrosis (Grades MF-0, MF-1+, MF-2-3+), and cytogenetics. The following clinical data were gathered: age, gender, smoking history (never, former, or current), splenomegaly (Y/N), BMI $\left(\mathrm{kg} / \mathrm{m}^{2}\right)$, history of OSA $(\mathrm{Y} / \mathrm{N})$, treatment with phlebotomy $(\mathrm{Y} / \mathrm{N})$, and survival (alive or date of death).

Two hematopathologists independently reviewed the bone marrow without knowledge of patient age or category assignment and recorded the following information: evaluable biopsy length, cellularity in 10\% increments (if biopsy inadequate, clot sections used if adequate), megakaryocyte morphology (increased: $\mathrm{Y} / \mathrm{N}$; abnormal: $\mathrm{Y} / \mathrm{N}$; predominant type of abnormality: staghorn, cloud-like, or unclear), and reticulin fibrosis (WHO MF- 0,1 , or 2-3).

The following scheme for hypercellularity was adopted: age 18 -39 years, $\geq 70 \%$; 40-69 years, $\geq 60 \%$; and age $\geq 70$ years, $\geq 50 \% .^{13,14}$ The EPO method used in our laboratory was slightly modified in December 2013. Prior to that, the reference range was $4-24 \mathrm{mIU} / \mathrm{mL}$; subsequently it was 3-19 $\mathrm{mIU} / \mathrm{mL}$.

Mayo Clinic Laboratories performed the vast majority of JAK2 V617F testing for this study and reported a qualitative result prior to November 2011. Other reference laboratories gave a qualitative result throughout the study. Quantitative results were reported with an equivocal range of $0.06 \%-0.6 \%$.

Applying 2017 WHO criteria and subsequent clinical course, patients were placed into one of seven categories: PV, post-PV

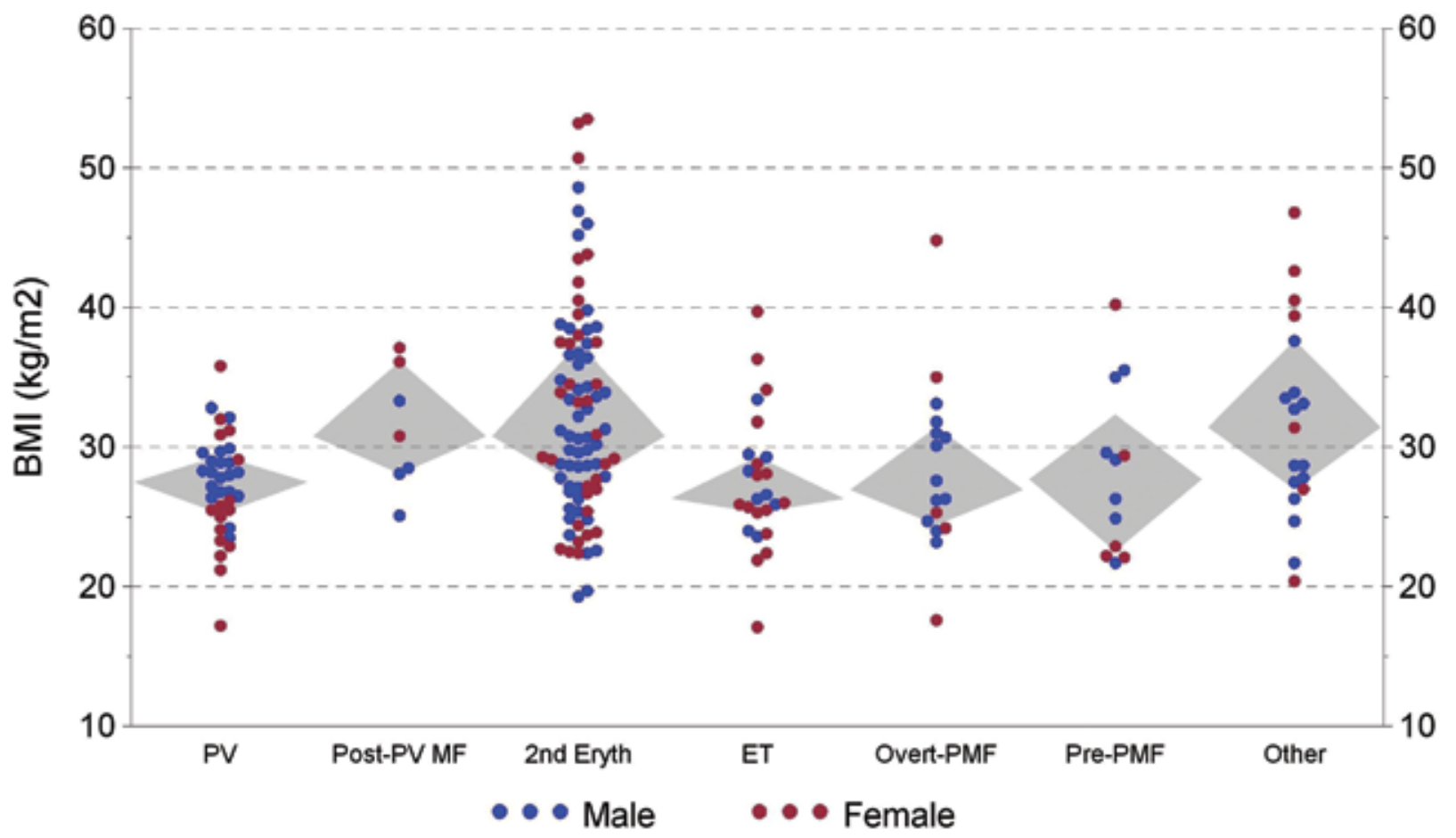

Figure 1. Patient BMI versus diagnostic category (Grey diamond-shaped areas encompass quartiles 2 and 3). 
Table 1 Patient characteristics and laboratory data by diagnostic group

\begin{tabular}{|c|c|c|c|c|c|c|c|}
\hline & PV & $\begin{array}{c}\text { Post-PV } \\
\text { MF }\end{array}$ & SE & ET & $\begin{array}{l}\text { Overt } \\
\text { PMF }\end{array}$ & PreMF & Other \\
\hline Total Patients & 36 & 7 & 85 & 25 & 16 & 12 & 19 \\
\hline Male (\%) & 55.6 & 57.1 & 60.0 & 36.0 & 68.8 & 58.3 & 63.2 \\
\hline Median age (yrs) & 73.3 & 66.7 & 57.5 & 73.5 & 72.5 & 79.9 & 56.4 \\
\hline Minimum & 23.7 & 59.4 & 16.3 & 22.8 & 44.6 & 50.2 & 26.5 \\
\hline Maximum & 88.6 & 86.3 & 86.2 & 92.3 & 90.3 & 97.1 & 82.1 \\
\hline Median BMI $\left(\mathrm{kg} / \mathrm{m}^{2}\right)$ & 27.5 & 30.8 & 30.8 & 26.3 & 27.0 & 27.7 & 31.4 \\
\hline Minimum & 17.2 & 25.1 & 19.3 & 17.1 & 17.6 & 21.7 & 20.4 \\
\hline Maximum & 35.8 & 37.1 & 53.5 & 39.7 & 44.8 & 40.2 & 46.8 \\
\hline Any smoking (\%) & 58.3 & 28.6 & 69.4 & 36.0 & 68.8 & 66.7 & 68.4 \\
\hline JAK2+ (\%) & 100 & 100 & 0.0 & 64.0 & 62.5 & 58.3 & 31.5 \\
\hline \multicolumn{8}{|l|}{$\mathrm{Hb}(\mathrm{g} / \mathrm{L})$ Females } \\
\hline Median & 172 & 153 & 176 & 142 & 145 & 149 & 138 \\
\hline Minimum & 142 & 139 & 129 & 131 & 111 & 114 & 115 \\
\hline Maximum & 206 & 183 & 203 & 158 & 151 & 159 & 158 \\
\hline \multicolumn{8}{|l|}{$\mathrm{Hb}(\mathrm{g} / \mathrm{L})$ Males } \\
\hline Median & 181 & 191 & 187 & 166 & 157 & 159 & 161 \\
\hline Minimum & 142 & 170 & 156 & 135 & 102 & 143 & 125 \\
\hline Maximum & 228 & 242 & 221 & 177 & 173 & 166 & 206 \\
\hline \multicolumn{8}{|c|}{$\mathrm{RBC}\left(\mathrm{x} 10^{6} / \mu \mathrm{L}\right)$ Females } \\
\hline Median & 6.0 & 5.3 & 5.6 & 4.8 & 5.0 & 4.6 & 4.8 \\
\hline Minimum & 4.7 & 4.4 & 5.0 & 4.4 & 3.3 & 3.8 & 4.0 \\
\hline Maximum & 10.3 & 7.5 & 7.9 & 6.1 & 5.7 & 5.9 & 6.5 \\
\hline \multicolumn{8}{|l|}{ RBC $\left(x 10^{6} / \mu \mathrm{L}\right)$ Males } \\
\hline Median & 6.9 & 6.8 & 6.0 & 5.3 & 5.0 & 5.2 & 5.4 \\
\hline Minimum & 5.5 & 5.3 & 5.2 & 4.6 & 3.0 & 4.5 & 4.0 \\
\hline Maximum & 10.3 & 7.5 & 7.9 & 6.1 & 5.7 & 5.9 & 6.5 \\
\hline \multicolumn{8}{|l|}{ Platelets $\left(\times 10^{3} / \mu \mathrm{L}\right)$} \\
\hline Median & 686.0 & 617.0 & 283.0 & 811.0 & 593.0 & 864.5 & 568.0 \\
\hline Minimum & 164.0 & 145.0 & 121.0 & 414.0 & 123.0 & 247.0 & 169.0 \\
\hline Maximum & 1598.0 & 1501.0 & 757.0 & 1422.0 & 2437.0 & 1747.0 & 1067.0 \\
\hline \multicolumn{8}{|l|}{ MCV (fL) } \\
\hline Median & 84.2 & 76.2 & 89.1 & 87.7 & 84.7 & 87.8 & 86.8 \\
\hline Minimum & 55.6 & 65.8 & 64.3 & 80.9 & 71.5 & 82.4 & 74.4 \\
\hline Maximum & 94.5 & 86.6 & 102.0 & 93.4 & 97.4 & 95.7 & 91.9 \\
\hline \multicolumn{8}{|l|}{ EPO (mlU/mL) } \\
\hline Median & 2.5 & 4.0 & 7.3 & 5.1 & 13.3 & 18.0 & 14.3 \\
\hline Minimum & 0.6 & 1.5 & 1.0 & 5.0 & 5.0 & 18.0 & 4.0 \\
\hline Maximum & 14.0 & 4.0 & 58.0 & 8.0 & 100.0 & 18.0 & 132.0 \\
\hline
\end{tabular}

$\mathrm{BMI}$, body mass index; $\mathrm{Hb}$, hemoglobin; EPO, erythropoietin; MCV, mean corpuscular volume; RBC, red blood cell; $\mathrm{PV}$, polycythemia vera; MF, myelofibrosis; SE, secondary erythrocytosis; $\mathrm{ET}$, essential thrombocythemia; PMF, primary myelofibrosis; PrePMF, prefibrotic primary myelofibrosis 


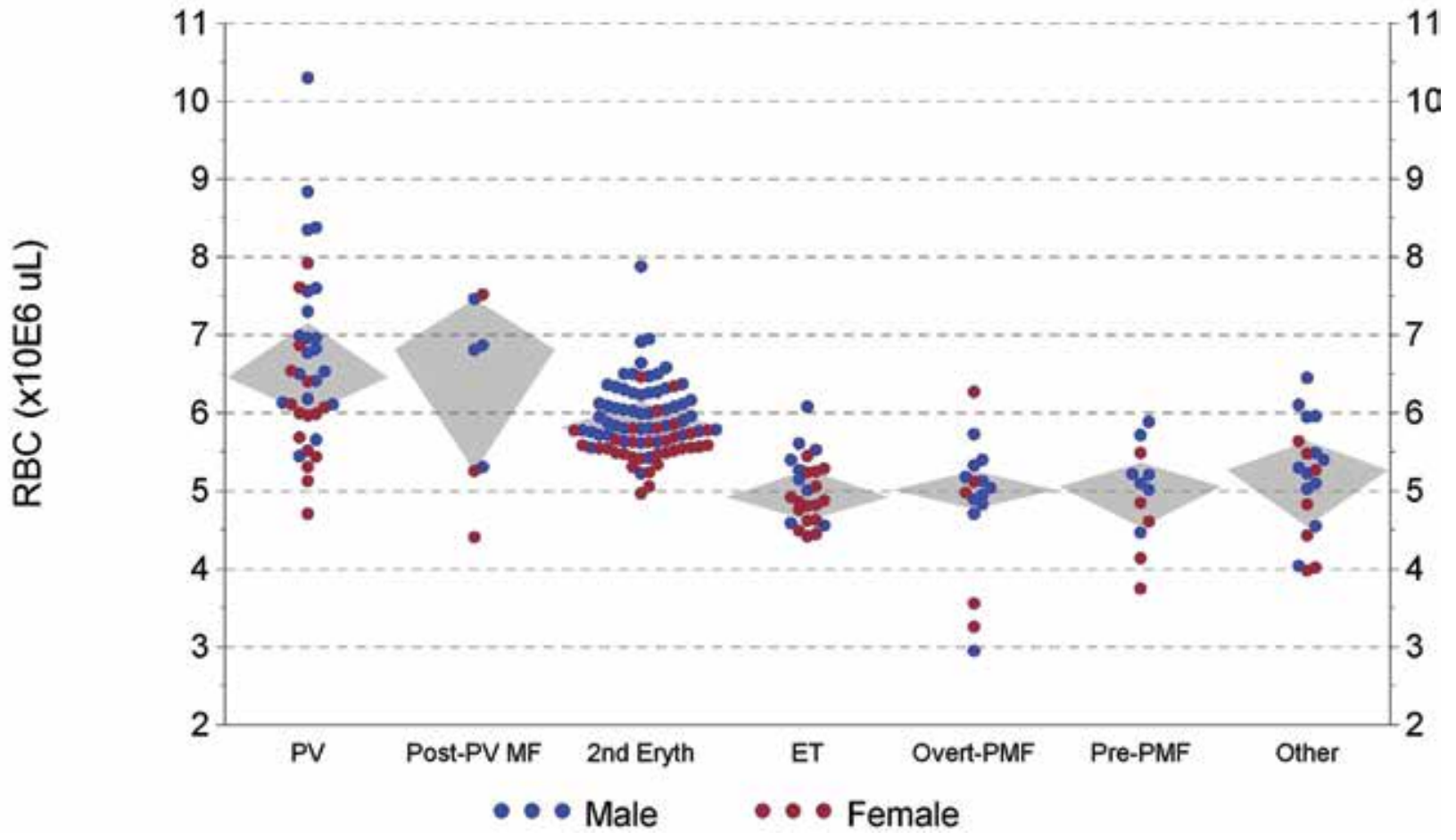

Figure 2. Highest previous red blood cell count versus diagnostic category (Grey diamond-shaped areas encompass quartiles 2 and 3).

myelofibrosis (post-PV MF), SE, essential thrombocythemia (ET), prefibrotic primary myelofibrosis (prePMF), overt PMF, or "other". The JAK2 V617F mutational burden was not used in category assignment.

Statistical comparisons were based on standard methods, including chi-square tests for categorical outcomes (eg, smoking status) and the Kruskal-Wallis test for continuous outcomes (eg, lab results). Since we did not have specific a priori hypotheses, and since we had no gold-standard disease classification, the results will require confirmation through additional studies.

\section{Results}

For all patients in the Marshfield Clinic system who had a $\mathrm{Hb}$ level measured during 2016, the first value was $>165 \mathrm{~g} / \mathrm{L}$ in $7.7 \%$ of 62,000 men and $>160 \mathrm{~g} / \mathrm{L}$ in $1.4 \%$ of 84,000 women. In contrast, using the previous, more stringent $2008 \mathrm{WHO}$ criteria, only $0.3 \%$ of men had $\mathrm{Hb}>185 \mathrm{~g} / \mathrm{L}$, while $0.6 \%$ of women had $\mathrm{Hb}>165 \mathrm{~g} / \mathrm{L}$.

Table 1 summarizes the clinical and laboratory data for the 200 patients in this study. The seven patients classified as post-PV MF were similar to the other PV patients; thus, they were combined for statistical analyses. Patients with SE, as expected, had a lower mean age. The only category with more females than males was ET. Of SE patients, 45 of $85(53 \%)$ were current smokers versus 6 of $43 \mathrm{PV} /$ post-PV MF patients $(14 \%, P<0.001)$. Only 3 of 96 MPN patients were under 40 years-of-age.
Of SE patients, $46(54 \%)$ were obese (BMI $\left.>30 \mathrm{~kg} / \mathrm{m}^{2}\right)$ compared with $10(23 \%)$ of $43 \mathrm{PV} /$ post-PV MF patients $(P=$ $0.001)$ as shown in Figure 1. Sixteen of $19 \mathrm{SE}$ patients with a history of OSA were obese (BMI $>30 \mathrm{~kg} / \mathrm{m}^{2}$ ). Regarding obesity and cigarette smoking (either former or current) as risk factors for SE, 79 of $85(93 \%)$ SE patients had at least one risk factor, compared with 27 of 43 (63\%) PV/post-PV MF patients $(P<0.001)$.

$\mathrm{Hb}$ level was not helpful in distinguishing $\mathrm{PV} /$ post-PV MF from SE. Of the $43 \mathrm{PV} /$ post-PV MF patients, 25 met the 2008 WHO Hb criteria. Another 11 men met only the 2017 criteria; 8 had a BM evaluation, and all were hypercellular. Three did not have a BM evaluation, but their further clinical course supported PV as the correct assignment.

Seven additional patients (one male and six females) were classified as PV/post-PV MF despite all prior available $\mathrm{Hb}$ levels being $\leq 165 \mathrm{~g} / \mathrm{L}$ or $160 \mathrm{~g} / \mathrm{L}$, respectively. All seven had an elevated RBC and evidence of iron deficiency, with further clinical course supporting PV. Of 53 patients with ET or PMF, 8 (all men) had $\mathrm{Hb}$ levels above the 2017 WHO PV criterion, but this was felt to be SE superimposed upon their primary MPN.

Figure 2 shows $\mathrm{RBC}$ versus diagnostic group. Of 24 male PV/ post-PV MF patients, 14 had an RBC count $>6.8 \times 10^{6} / \mathrm{L}$ versus only 3 of $51 \mathrm{SE}$ patients $(P<0.001)$. For females, 11 of $19 \mathrm{PV} /$ post-PV MF patients had an RBC count $>5.9 \mathrm{x}$ $10^{6} / \mathrm{L}$ versus 3 of 33 SE patients $(P<0.001)$. 
Figure 3 shows platelet count versus diagnostic group. SE patients rarely had platelet counts $\geq 450,000 / \mu \mathrm{L}$ vs. patients with MPN $(P<0.001)$.

The MCV and ferritin levels trended lower in the PV/post-PV MF patients, but with overlap. The $\mathrm{MCV} / \mathrm{RBC}$ ratio for each patient in the various categories did not show better group separation.

As shown in Figure 4, EPO levels were usually lower in PV/ post-PV MF patients vs. SE, but with overlap. Of $27 \mathrm{PV} /$ postPV MF patients, 20 had low EPO levels versus 15 of $72 \mathrm{SE}$ patients $(P<0.001)$. Levels above $9 \mathrm{mIU} / \mathrm{mL}$ were common in SE patients, but were seen in just one PV patient when the $\mathrm{Hb}$ level was $144 \mathrm{~g} / \mathrm{L}$ from blood loss.

All $43 \mathrm{PV} /$ post-PV MF patients were JAK2+, with just one having an exon 12 mutation. He had a classic PV clinical presentation and clinical course. For ET, prePMF, and overt PMF, a slight majority $(16 / 25,10 / 16$, and $7 / 12$, respectively) were JAK2 $\mathrm{V} 617 \mathrm{~F}+$.

Among 19 patients classified as "other" were four patients with myelodysplastic /myeloproliferative neoplasm with ring sideroblasts and thrombocytosis (MDS/MPN-RS-T). Another four had an unclassifiable MDS or MPN. One patient had congenital erythrocytosis. Another had a presentation like PV with low EPO, but was negative for JAK2 V617F and exon 12 mutations. Another patient was favored to have SE, but had equivocal JAK2 V617F results. Lastly, eight patients likely had a reactive cause for their elevated counts.

Eight patients had JAK2 V617F values in the equivocal range, but follow-up testing did not show an upward trend, and none were diagnosed with an MPN. Five were classified as SE based on other laboratory data and clinical presentation. One PV patient initially had a false negative JAK2 V617F test by an allele-specific assay. Subsequent sequencing actually found the V617F mutation along with a second mutation in exon 14 that likely disrupted primer binding.

Cytogenetic testing was performed on 112 patients with an available BM aspirate, and 17 had cytogenetic abnormalities associated with a myeloid neoplasm. A 20q deletion was the most common abnormality, seen in seven cases. Eight of 21 patients with MPN having myelofibrosis (combining post-PV MF and overt PMF) had abnormal cytogenetics.

Three PV/post-PV MF patients went on to develop acute myeloid leukemia, and one developed a high grade myeloid neoplasm with 14\% BM blasts. One overt PMF patient ("triple negative" for JAK2V617F, CALR, and MPL) developed acute myeloid leukemia.

Of nine JAK2 V617F-negative ET patients, eight were tested for a CALR mutation, and two were positive. One ET patient had a myeloproliferative leukemia (MPL) Trp515Leu mutation. Of 11 prePMF or overt PMF patients that were

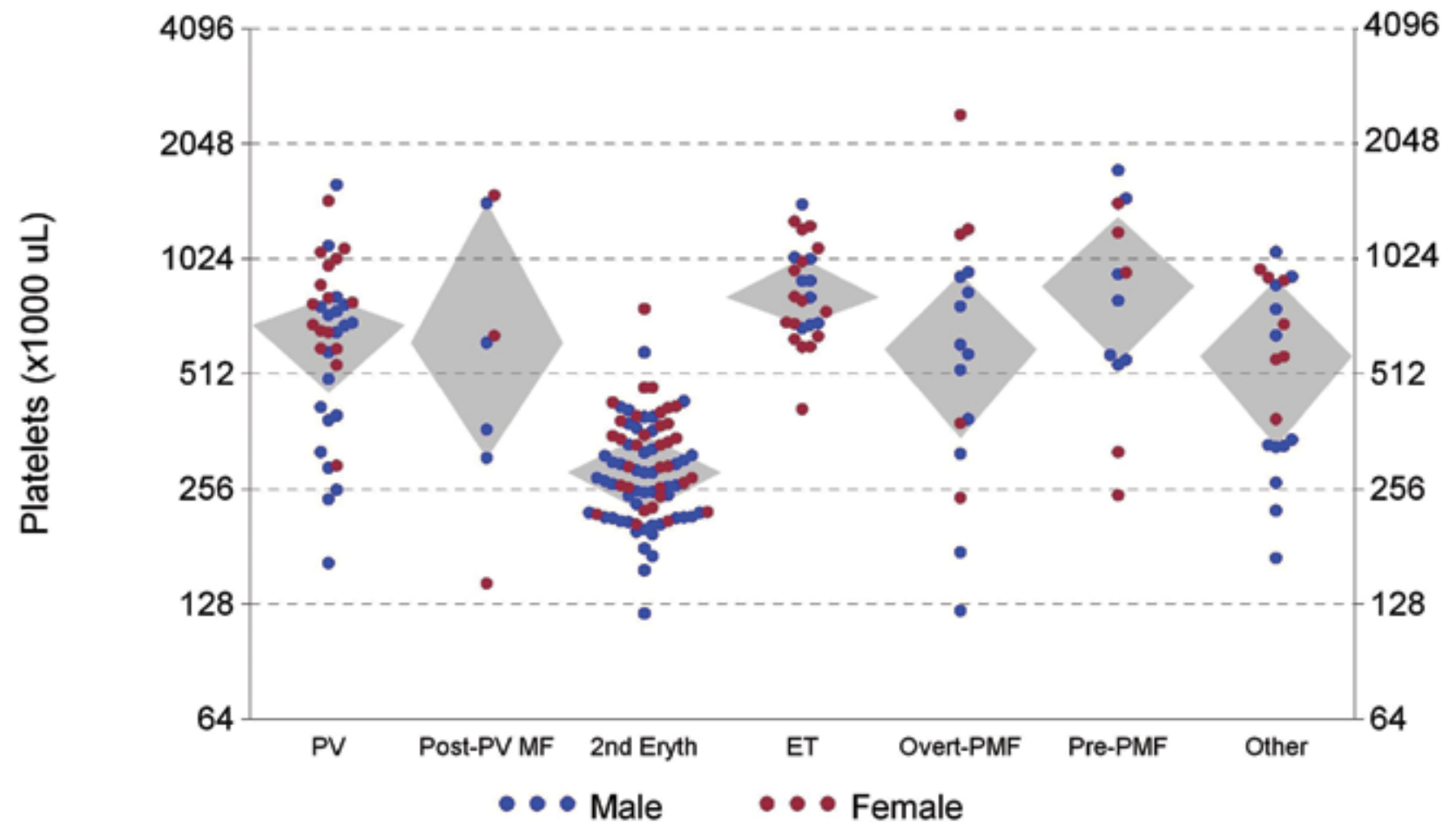

Figure 3. Highest previous platelet count versus diagnostic category (Grey diamond-shaped areas encompass quartiles 2 and 3 ). 
JAK2 V617F-negative, only five had CALR testing, and three were mutated.

Very few patients had red cell mass measurements. One patient was erroneously considered Gaisbock syndrome (erythrocytosis putatively due to plasma volume contraction) based on a normal red cell mass. Eventually he was diagnosed with PV, confirmed by a JAK2 V617F mutation.

There were $111 \mathrm{BM}$ specimens available for review. In 13 cases, there was either no biopsy or it was of inadequate length $(\leq 0.2 \mathrm{~cm}$ ). Of the remaining 98 biopsies, 61 were $0.3-0.9 \mathrm{~cm}$ in evaluable length, 28 were $1.0-1.4 \mathrm{~cm}$, and $9 \mathrm{had}$ the WHO recommended $\geq 1.5 \mathrm{~cm} .15$ For reticulin fibrosis, 18 of 91 cases that could be assessed were called MF- 0 or MF-1+ by one hematopathologist, but MF-2-3+ by the other, which could influence diagnostic category assignment. The subjective assessment of megakaryocytes as having "staghorn" or "cloudlike" nuclei showed poor concordance.

Of 43 patients with PV/post-PV MF, 31 had adequate BM samples to evaluate cellularity. In 28 patients, it was interpreted as hypercellular by our hematopathologists. Megakaryocytes were felt to be increased in all PV/post-PV MF patients and abnormal in all but two. Of the 85 SE patients, 26 underwent $\mathrm{BM}$ evaluation. BM was called hypercellular in just three patients $(P<0.001$ when compared with $\mathrm{PV} /$ post-PV MF.

Of 16 patients with overt PMF, 15 had BM evaluation, with hypercellularity for age in 13 . Four of the 16 had a WBC $\geq 11$ $\mathrm{x} 10^{3} / \mu \mathrm{L}$. For prePMF, 11 of 12 had BM evaluation, with nine being hypercellular. Nine of the 12 patients had a WBC count $\geq 11 \times 10^{3} / \mu \mathrm{L}$.

Of 25 ET patients, 18 had a BM evaluation; three were slightly hypercellular for age. Six of the 25 had a WBC $\geq 11 \times 10^{3} / \mu \mathrm{L}$. None of 25 ET patients had splenomegaly, versus 5 of 12 prePMF patients.

Based on visual inspection of the scatter plots and statistical comparisons cited above, threshold values were chosen to assist in differentiating PV from SE. RBC $>6.8 \times 10^{6} / \mu \mathrm{L}$ for men or $>5.910^{6} / \mu \mathrm{L}$ for women, platelets $\geq 450 \times 10^{3} / \mu \mathrm{L}$, EPO $<4 \mathrm{mIU} / \mathrm{mL}$, and $\mathrm{MCV}<80 \mathrm{fL}$ (or ferritin $<24 \mathrm{ng} / \mathrm{mL}$ ) increased the probability of PV vs. SE. Age $<40$ years, BMI $>30 \mathrm{~kg} / \mathrm{m}^{2}$ (and especially $>35 \mathrm{~kg} / \mathrm{m}^{2}$ ), and EPO $>9 \mathrm{mIU} / \mathrm{mL}$ reduced the probability.

The following PV risk scoring system was devised as an exploratory investigation:

Age $<40$ years: -1

$\mathrm{RBC}>6.8 \times 10^{6} / \mathrm{uL}$ (men) or $>5.9 \times 10^{6} / \mathrm{uL}$ (women): +1 Platelets $\geq 45010^{3} / \mathrm{uL}:+1$

BMI $30-35 \mathrm{~kg} / \mathrm{m}^{2}:-0.5$

BMI $>35 \mathrm{~kg} / \mathrm{m}^{2}$ or history of OSA: -1

Smoking: currently or chronic obstructive pulmonary disease (COPD): -0.5

$\mathrm{MCV}<80 \mathrm{fL}$ or ferritin $<24:+0.5$

EPO* $<4 \mathrm{mIU} / \mathrm{mL}:+0.5$

$\mathrm{EPO}^{*}>9 \mathrm{mIU} / \mathrm{mL}:-1$

*EPO is often not available at initial patient presentation.

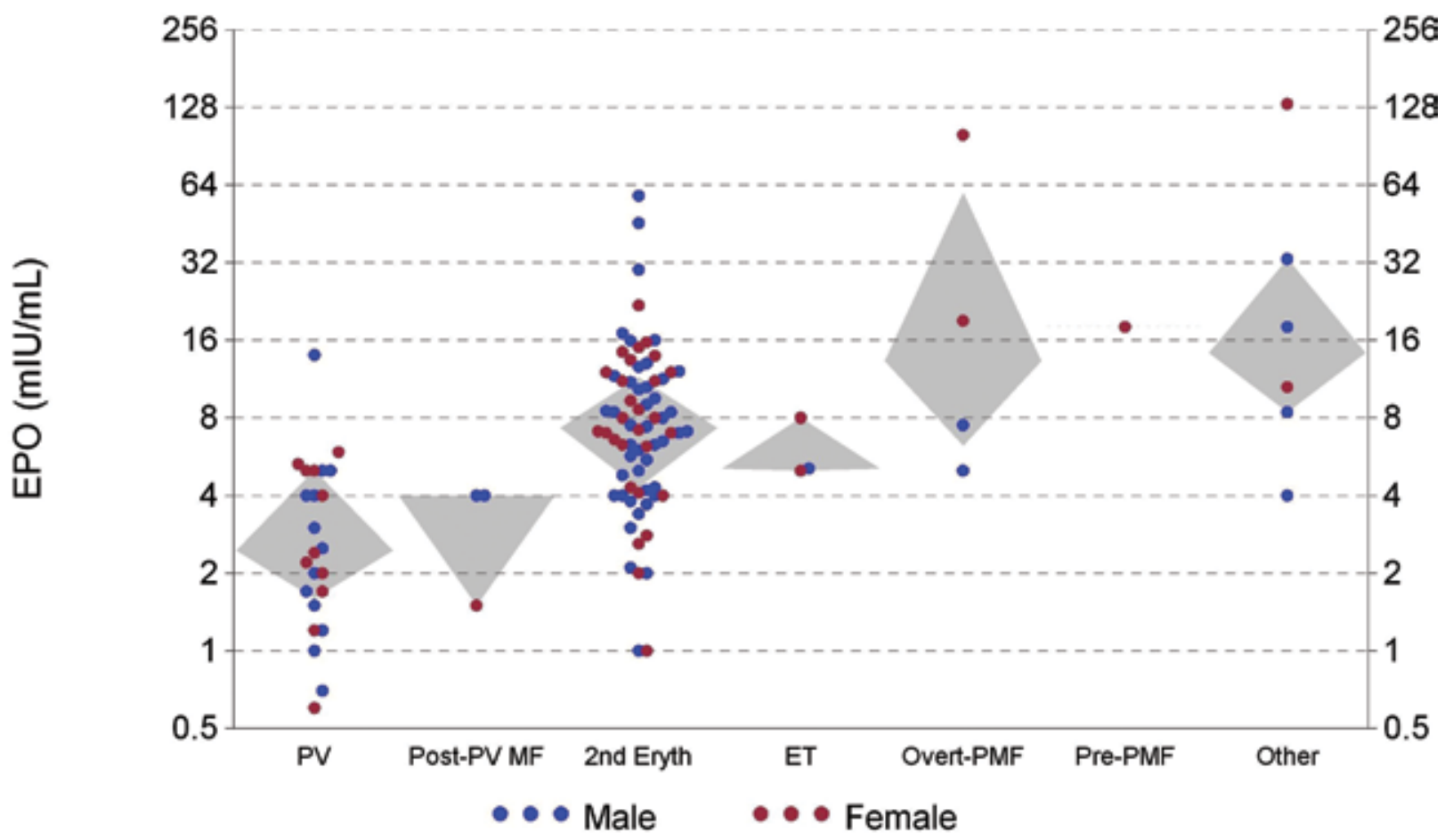

Figure 4. Erythropoietin levels versus diagnostic category. (Grey diamond-shaped areas encompass quartiles 2 and 3. ) 


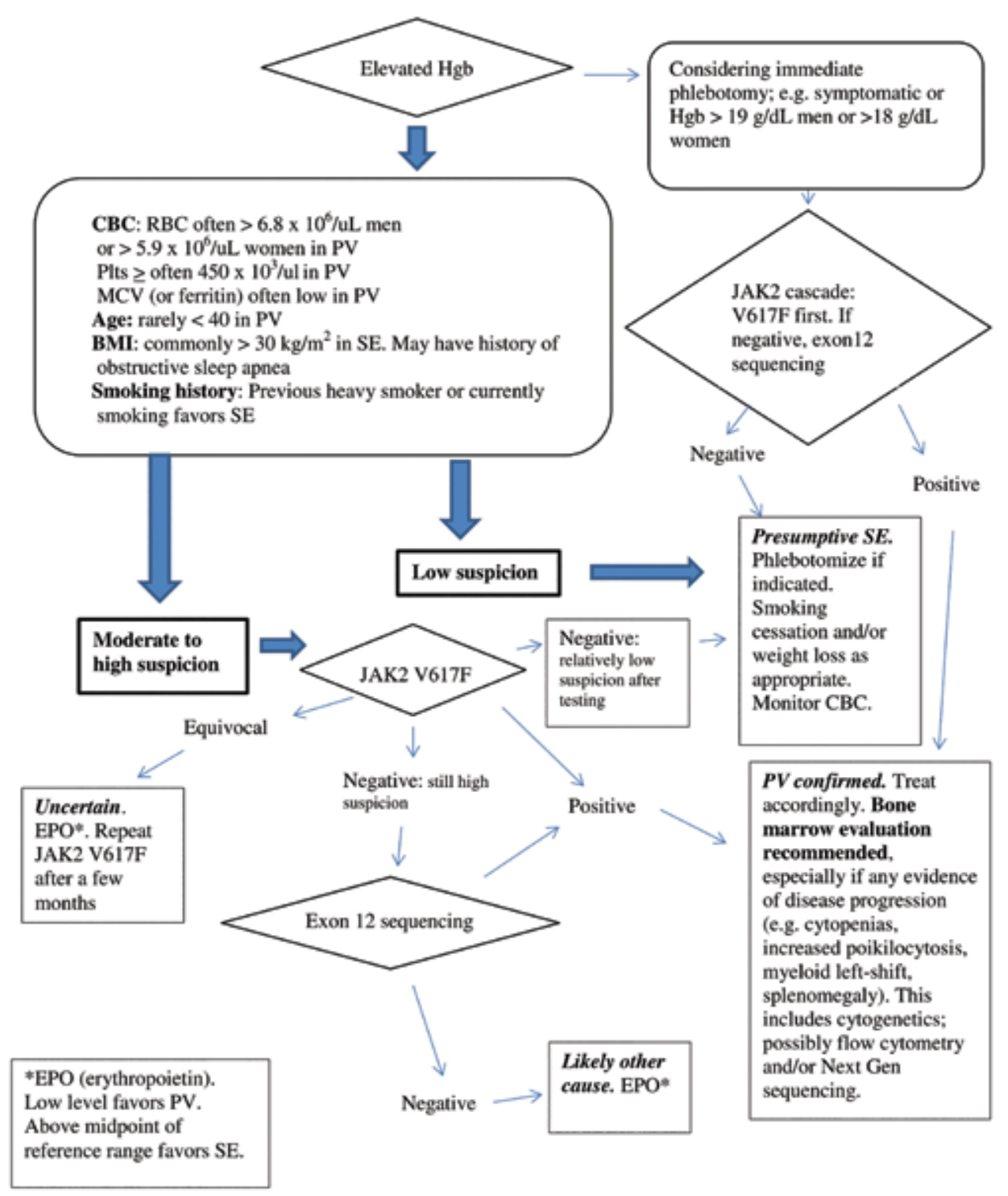

Figure 5. Suggested algorithm for working up possible polycythemia vera vs. secondary erythrocytosis.

Without EPO, a total score of $\geq 0.5$ yielded a sensitivity of $93 \%$ and specificity of $94 \%$ for diagnosing PV. Incorporating EPO levels improved both sensitivity and specificity to $98 \%$ using the same total score cutoff.

\section{Discussion}

The prevalence of PV in the United States was estimated at 22 in 100,000 individuals in $2008 .{ }^{16}$ Based on the $\mathrm{Hb}$ data in the Marshfield Clinic system cited above, roughly 1 in 350 males and 1 in 64 females with $\mathrm{Hb}>165 \mathrm{~g} / \mathrm{L}$ or $>160 \mathrm{~g} / \mathrm{L}$, respectively, would be expected to have PV. Thus, use of the new $\mathrm{Hb}$ criteria to trigger JAK2 testing, without consideration of other clinical factors, could result in excessive testing. ${ }^{7,8,17}$

In this study, $74 \%$ of PV patients had a low EPO level, which corresponds with a recent review. ${ }^{18}$ However, the specificity (when applied to SE patients) was $79 \%$, which is considerably below the $92 \%-99 \%$ cited in that review. In our study, a few
PV patients were identified who had high EPO levels during a time of transient blood loss, which is an appropriate physiologic response. Similarly, patients who undergo therapeutic phlebotomy will eventually raise their EPO levels provided renal function is normal. ${ }^{19}$ Therefore, EPO levels need to be obtained when the $\mathrm{Hb}$ level is at least high-normal.

The RBC count is a useful parameter in separating PV from SE. ${ }^{9}$ In our study, about $60 \%$ of PV patients had values $>6.8$ x $10^{6} / \mu \mathrm{L}$ for males and $>5.9 \times 10^{6} / \mu \mathrm{L}$ for females, whereas few SE patients exceeded these cutoffs (Figure 2). Although, thalassemia often has a high $\mathrm{RBC}$ and low $\mathrm{MCV}$, in clinical practice this rarely poses a problem. Thalassemia is a genetic disorder characterized by a lifelong high RBC and low MCV without an elevated $\mathrm{Hb}$ level. ${ }^{20}$

Many laboratories have an equivocal range for the JAK2 V617F mutation. Values in this range should be repeated at a 
later date. A Danish study found JAK2 V617F mutations in 63 of $49,488(0.1 \%)$ of unselected individuals. ${ }^{21}$ Patients in their equivocal range later manifesting clinical disease showed an upward trend.

Whether true JAK2-negative PV exists is controversial. ${ }^{22,23}$ However, since only about $2 \%$ of PV patients have an exon 12 mutation, it is debatable whether all JAK2 V617F-negative patients being evaluated for PV should have this as a followup test.

As a criterion for MPN diagnosis, BM morphology presents several challenges. Well-validated age-adjusted cutoffs for BM cellularity have not been established to diagnose MPN. ${ }^{13,14}$ The current study used a simple scheme for age-adjusted hypercellularity that could be adopted in routine practice. Reactive/regenerative changes can also increase BM cellularity. Furthermore, the biopsy may be small with crush artifact, procedure-related hemorrhage, or have hypocellular subcortical areas not apparent from the biopsy orientation.

In our study with limited morphologic evaluation, the subtyping of megakaryocytes (ie, staghorn or cloud-like) was unreliable. ${ }^{24}$ Thus, the distinction between ET and prePMF was essentially based on BM cellularity, WBC count, and splenomegaly; M:E ratio was not helpful. A recent study of 211 BM biopsies independently reviewed by two hematopathologists found low reproducibility of the WHO histologic criteria for MPN, even though all biopsies were $\geq$ $1.5 \mathrm{~cm}$ in length. 25

Cellularity is best evaluated from the biopsy, ideally $\geq 1.5 \mathrm{~cm}$ in length without crush artifact ${ }^{15}$; however, in many practice settings biopsies are usually smaller. ${ }^{26}$ Clot sections are suboptimal, but can be used if they have moderately sized spicules $\geq 0.2 \mathrm{~cm}$. Aspirate smears can only give a qualitative estimate.

In the appropriate clinical context, an MPN "driver mutation" (ie, JAK2, CALR, or MPL) establishes the presence of a myeloid neoplasm. ${ }^{23} \mathrm{BM}$ evaluation may yield important prognostic information; e.g. percentage of marrow blasts, dysplasia, ring sideroblasts, and marrow fibrosis. Also, BM generally provides the best sample for a cytogenetic study.

Some hematologists may be reluctant to embrace BM evaluation as a mandatory major criterion for PV diagnosis. For example, in a JAK2 $\mathrm{V} 617 \mathrm{~F}+$ patient with an elevated $\mathrm{Hb}$ and no reason for $\mathrm{SE}$, a diagnosis of $\mathrm{PV}$ is reasonably secure without BM evaluation. However, the presence of increased poikilocytosis (including nucleated RBCs or teardrop forms), a myeloid left-shift (possibly including circulating blasts), or splenomegaly would raise concern of myelofibrosis or disease progression, and bone marrow evaluation would be clearly indicated.
Conventional cytogenetics continue to play a key role in the diagnosis and prognostication of myeloid neoplasms. ${ }^{27,28}$ The finding of relatively frequent cytogenetic abnormalities in MPN patients with marrow fibrosis (overt PMF and post-PV MF) fits with the observation that these patients have greater overall genetic complexity contributing to their unfavorable prognosis. $^{29-31}$

When facing a differential diagnosis of PV vs. SE, the simple scoring system provided in this study may assist in deciding when to proceed to further diagnostic testing. Relevant clinical and laboratory information available in nearly all patients includes age, gender, $\mathrm{RBC}$, platelet count, $\mathrm{MCV}$, and BMI. Smoking history has limited reliability, but current smoking or a history of heavy smoking (often accompanied by COPD) is helpful. Although a history of OSA may provide additional support for SE (beyond obesity), it is often not well documented nor is its severity quantified. In contrast, BMI is more objective and increasingly documented in the medical record. Ferritin level is occasionally available and augments MCV in assessing iron deficiency.

A suggested diagnostic algorithm is presented in Figure 5 to differentiate PV from SE. A more formal PV-risk scoring system would require larger patient cohorts to perform multivariate analysis with assignment of optimal cutoffs and weighting of risk factors.

Costs of laboratory tests vary widely between individuals, depending on payer (private insurance or government) and discounts. Currently within the Marshfield Clinic Health System, JAK2V617F, JAK2 exon 12, and EPO tests have comparable fees, roughly between $\$ 150$ and $\$ 200$.

Our study reflects real-life clinical practice and the everyday challenges in diagnosing MPN. Limitations include patient selection that was not done in a way to provide a statistically representative sample, and patients were diagnosed at different times in the course of their disease. The small size of the subgroups limited the power of statistical comparisons. Genetic data, other than JAK2 V617F, was sporadically available. The patients were not uniformly treated, and $42 \%$ did not have a BM evaluation. MPL and CALR tests, which became more widely available in 2009 and early 2014, respectively, were done in a relatively small number of cases.

Our patient cohort was predominantly of northern European descent from the upper Midwest of the United States at about 360 meters above sea level. Other populations may differ significantly in prevalence of obesity or cigarette smoking. Therefore, the use of these clinical parameters for differentiating PV from SE may need modification in other settings.

JAK2+ MPN may have overlapping phenotypes that evolve over time. ${ }^{27}$ In this study, several JAK2 V617F+ patients 
were difficult to classify due to the possibility of superimposed SE; a few evolved to a clear-cut PV presentation. The phenotype and prognosis is influenced by the mutational burden and additional genetic aberrations that are increasingly being elucidated by gene panel sequencing. ${ }^{32-36}$

This study was conducted using the classical paradigm for non-chronic myeloid leukemia MPN, attempting to classify each patient as either PV, post-PV MF, ET, prePMF, or overt PMF. Looking to the future, with advances in our understanding of the molecular basis of MPN, this scheme may need revision. A new model (similar to that for acute leukemias) could primarily subclassify these disorders by their driver mutation(s) and other genetic abnormalities. ${ }^{37}$

Various lines of evidence support such a change. Rather arbitrary $\mathrm{Hb}$ levels have been proposed to discriminate "masked" PV from JAK2+ ET. ${ }^{27}$ Yet, nearly $30 \%$ of patients presenting as JAK2+ ET progress to a PV phenotype at 15 years. ${ }^{38}$ Approximately $10 \%$ of PV and 5\% of ET cases develop myelofibrosis indistinguishable from overt PMF at 15 years. ${ }^{39}$ The distinction between this evolution and overt PMF presumably evolved from prePMF is not possible if there is inadequate historical data. Moreover, such separation does not influence patient management. PMF patients with CALR mutations (mostly the more favorable type 1 with a $52 \mathrm{BP}$ deletion) have a better prognosis than JAK2 V617F+ patients, who in turn do better than "triple negative" cases. ${ }^{40}$ Mutational status acquired by sequencing a panel of relevant genes may become the best predictor of future disease course and response to treatment. ${ }^{37,41}$

\section{Conclusion}

In summary, this study of 200 patients who underwent JAK2 V617F mutation testing because of a clinically suspected MPN provided several key findings, which will require confirmation by future studies.

1. The WHO $2017 \mathrm{Hb}$ diagnostic criteria were not intended to be used indiscriminately to screen for PV. Hb level alone is not helpful in distinguishing PV from SE. However, PV patients often have higher RBC values $\left(>6.8 \times 10^{6} / \mu \mathrm{L}\right.$ for men and $>5.9 \times 10^{6} / \mu \mathrm{L}$ for women) along with lower MCV and ferritin values than patients with SE. Platelet counts $\geq$ $450,000 / \mu \mathrm{L}$ are rare in SE patients.

2. The decision to "trigger" molecular testing and/or BM evaluation should incorporate clinical information. MPN patients are rarely under age 40 (only 3\% in this study). The vast majority of SE patients (93\% in this study) have at least one risk factor (eg, obesity $\left[\mathrm{BMI} \geq 30 \mathrm{~kg} / \mathrm{m}^{2}\right.$ ] or smoking history.)

3. JAK2 V617F positivity (above each laboratory's equivocal range) is diagnostic of MPN in the appropriate clinical context. However, equivocal values are not confirmatory and repeat testing is advised.

4. Although low EPO levels are more common in PV, this finding is not specific enough to confirm the diagnosis. Conversely, high EPO values above the reference range midpoint support SE, provided the Hb level is at least highnormal at the time of testing.

5. Currently, JAK2 exon 12 sequencing can be reserved for selected cases, rather than being a reflex order upon negative JAK2 V617F testing. This recommendation may change if costs come down.

6. BM evaluation (including a biopsy of adequate size) can assess marrow blasts, myelofibrosis, and provide a sample for cytogenetics. It may assist in distinguishing classical MPN subtypes and should be performed before therapeutic intervention.

\section{Acknowledgements}

The authors acknowledge the expert assistance of Kajal Sitwala, MD, PhD, Department of Lab/Pathology, Marshfield Clinic, for assistance with this project; and Marie Fleisner, Editorial Specialist, Marshfield Clinic Research Institute, for assistance in preparing the manuscript for publication.

\section{References}

1. Arber DA, Orazi A, Hasserjian R, et al. The 2016 revision to the World Health Organization classification of myeloid neoplasms and acute leukemia. Blood. 2016;127(20):23912405.

2. WHO Classification of Tumours of Haemotopoietic and Lympoid Tissues. Fifth Edition. Swerdlow SH, Campo E, Harris NL, et al., eds. Geneva, Switzerland: WHO Press; 2017.

3. Barbui T, Thiele J, Carobbio A, et al. Masked polycythemia vera diagnosed according to WHO and BCSH classification. Am J Hematol. 2014;89(2):199-202.

4. Gianelli U, Iurlo A, Vener C, et al. The significance of bone marrow biopsy and JAK2V617F mutation in the differential diagnosis between the "early" prepolycythemic phase of polycythemia vera and essential thrombocythemia. Am J Clin Pathol. 2008;130(3):336-342.

5. Barbui T, Thiele J, Vannucchi AM, Tefferi A. Rationale for revision and proposed changes of the WHO diagnostic criteria for polycythemia vera, essential thrombocythemia and primary myelofibrosis. Blood Cancer J. 2015;5(8):e337.

6. Barbui T, Thiele J, Gisslinger H, Carobbio A, Vannucchi AM, Tefferi A. Diagnostic impact of the 2016 revised who criteria for polycythemia vera. Am J Hematol. 2017;92(5):417-419.

7. Ethier V, Sirhan S, Olney HJ, et al. The 2016 WHO criteria for the diagnosis of polycythemia vera: benefits and potential risks. Blood. 2016;127:2391-2405. [Letter] Available at: http://www.bloodjournal.org/content/127/20/2391/tab-eletters.

8. Busque L, Porwit A, Day R, et al. Laboratory investigation of myeloproliferative neoplasms (MPNs): recommendations of the Canadian MPN Group. Am J Clin Pathol. 2016;146(4):408-422.

9. Usman M, Bilwani F, Kakepoto GN, Adil SN, Sajid R, Khurshid M. Polycythemia vera and idiopathic erythrocytosis: comparison of clinical and laboratory parameters. J Pak Med Assoc. 2004;54(5):249-251.

10. Kambali S, Taj A. Polycythemia vera masked due to severe iron deficiency anemia. Hematol Oncol Stem Cell Ther. 2018;11(1):38-40.

11. Mossuz P, Girodon F, Donnard M, et al. Diagnostic value of serum erythropoietin level in patients with absolute erythrocytosis. Haematologica. 2004;89(10):1194-1198. 
12. Goldman JJ, Vandris K, Adriano F, et al. Normal serumerythropoietin (S-epo) level at diagnosis of polycythemia vera (PV) correlates with low JAK2 V617F mutant allele burden and indicates mild phenotype. Blood. 2009;114:4978. abstract.

13. Hartsock RJ, Smith EB, Petty CS. Normal variation with aging of the amount of hematopoietic tissue in bone marrow from the anterior iliac crest. A study made from 117 cases of sudden death examined by necropsy. Am J Clin Pathol. 1965;43(4):326-331.

14. Thiele J, Kvasnicka HM, Facchetti F, Franco V, van der Walt J, Orazi A. European consensus on grading bone marrow fibrosis and assessment of cellularity. Haematologica. 2005;90(8):1128-1132.

15. Dayton VJ, Fink J, Linden MA, et al. Quality and adequacy of bone marrow samples obtained by the 2-needle technique: the Minnesota experience. Arch Pathol Lab Med. 2014;138(7):860-862.

16. Ma X, Vanasse G, Cartmel B, Wang Y, Selinger HA. Prevalence of polycythemia vera and essential thrombocythemia. Am J Hematol. 2008;83(5):359-362.

17. Horvitz RA. Diagnostic criteria for polycythemia vera: difference in changes between males and femailes. Am J Clin Pathol. 2017;147(4):439-440.

18. Tefferi A. Clinical manifestations and diagnosis of polycythemia vera. UpToDate. 2017. Available at: https://www.uptodate. $\mathrm{com} /$ contents/clinical-manifestations-and-diagnosis-ofpolycythemia-vera.

19. Artunc F, Risler T. Serum erythropoietin concentrations and responses to anaemia in patients with or without chronic kidney disease. Nephrol Dial Transplant. 2007;22(10):29002908.

20. Vehapoglu A, Ozgurhan G, Demir AD, et al. Hematological indices for differential diagnosis of Beta thalassemia trait and iron deficiency anemia. Anemia. 2014;2014:1-7.

21. Nielsen C, Bojesen SE, Nordestgaard BG, Kofoed KF, Birgens HS. JAK2V617F somatic mutation in the general population: myeloproliferative neoplasm development and progression rate. Haematologica. 2014;99(9):1448-1455.

22. Rumi E, Cazzola M. Diagnosis, risk stratification, and response evaluation in classical myeloproliferative neoplasms. Blood. 2017;129(6):680-692.

23. Tefferi A, Barbui T. Polycythemia vera and essential thrombocythemia: 2017 update on diagnosis, riskstratification, and management. Am J Hematol. 2017;92(1):94-108.

24. Jeryczynski G, Thiele J, Gisslinger B, et al. Pre-fibrotic/early primary myelofibrosis vs. WHO-defined essential thrombocythemia: The impact of minor clinical diagnostic criteria on the outcome of the disease. Am J Hematol. 2017;92(9):885-891.

25. Alvarez-Larrán A, Ancochea A, García M, et al. WHOhistological criteria for myeloproliferative neoplasms: reproducibility, diagnostic accuracy and correlation with gene mutations and clinical outcomes. Br J Haematol. 2014;166(6):911-919.

26. Merzianu M, Groman A, Hutson A, et al. Trends in Bone Marrow Sampling and Core Biopsy Specimen Adequacy in the United States and Canada. Am J Clin Pathol. 2018;150(5):393-405.

27. Reilly JT. Pathogenetic insight and prognostic information from standard and molecular cytogenetic studies in the BCR-ABLnegative myeloproliferative neoplasms (MPNs). Leukemia. 2008;22(10):1818-1827.

28. Vaidya R, Caramazza D, Begna KH, et al. Monosomal karyotype in primary myelofibrosis is detrimental to both overall and leukemia-free survival. Blood. 2011;117(21):5612-5615.
29. Nangalia J, Green TR. The evolving genomic landscape of myeloproliferative neoplasms. Hematology. 2014;2014(1):287-296.

30. Song J, Hussaini M, Zhang H, et al. Comparison of the mutational profiles of primary myelofibrosis, polycythemia vera, and essential thrombocytosis. Am J Clin Pathol. 2017;147(5):444-452.

31. Mora B, Giorgino T, Guglielmelli P, et al. Value of cytogenetic abnormalities in post-polycythemia vera and post-essential thrombocythemia myelofibrosis: a study of the MYSEC project. Haematologica. 2018;103(9):e392-e394.

32. Larsen TS, Pallisgaard N, Møller MB, Hasselbalch HC. The JAK2 V617F allele burden in essential thrombocythemia, polycythemia vera and primary myelofibrosis - impact on disease phenotype. Eur J Haematol. 2007;79(6):508-515.

33. Lundberg P, Karow A, Nienhold R, et al. Clonal evolution and clinical correlates of somatic mutations in myeloproliferative neoplasms. Blood. 2014;123(14):2220-2228.

34. Guglielmelli P, Lasho TL, Rotunno G, et al. The number of prognostically detrimental mutations and prognosis in primary myelofibrosis: an international study of 797 patients. Leukemia. 2014;28(9):1804-1810.

35. Tefferi A, Guglielmelli P, Larson DR, et al. Long-term survival and blast transformation in molecularly annotated essential thrombocythemia, polycythemia vera, and myelofibrosis. Blood. 2014;124(16):2507-2513, quiz 2615.

36. Tefferi A, Lasho TL, Guglielmelli P, et al. Targeted deep sequencing in polycythemia vera and essential thrombocythemia. Blood Adv. 2016;1(1):21-30.

37. Grinfeld J, Nangalia J, Baxter EJ, et al. Classification and Personalized Prognosis in Myeloproliferative Neoplasms. N Engl J Med. 2018;379(15):1416-1430.

38. Rumi E, Pietra D, Ferretti V, et al; Associazione Italiana per la Ricerca sul Cancro Gruppo Italiano Malattie Mieloproliferative Investigators. JAK2 or CALR mutation status defines subtypes of essential thrombocythemia with substantially different clinical course and outcomes. Blood. 2014;123(10):1544-1551.

39. Cerquozzi S, Tefferi A. Blast transformation and fibrotic progression in polycythemia vera and essential thrombocythemia: a literature review of incidence and risk factors. Blood Cancer J. 2015;5(11):e366.

40. Tefferi A, Lasho TL, Tischer A, et al. The prognostic advantage of calreticulin mutations in myelofibrosis might be confined to type 1 or type 1-like CALR variants. Blood. 2014;124(15):2465-2466.

41. Patel KP, Newberry KJ, Luthra R, et al. Correlation of mutation profile and response in patients with myelofibrosis treated with ruxolitinib. Blood. 2015;126(6):790-797.

\section{Author Affiliations}

Gene Shaw, MD* and Richard Berg, MS†

\author{
*Department of Lab-Pathology, Marshfield Clinic, \\ Marshfield, Wisconsin \\ †Biomedical Informatics Research Center, Marshfield Clinic \\ Research Institute, Marshfield, Wisconsin
}

